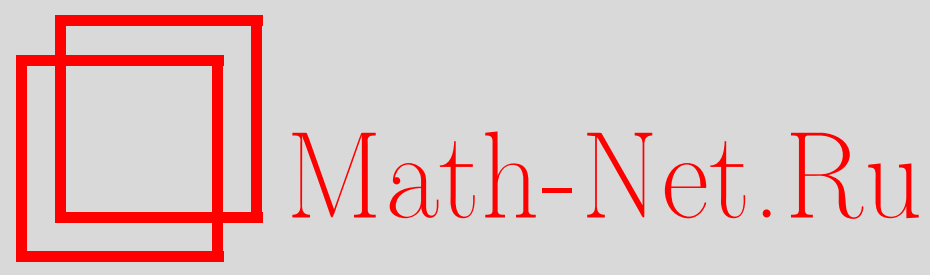

Б. З. Шаваровский, О разложимых многочленных матрицах, Матем. заметки, 2000, том 68, выпуск 4, 593-607

DOI: https://doi.org/10.4213/mzm980

Использование Общероссийского математического портала Math-Net.Ru подразумевает, что вы прочитали и согласны с пользовательским соглашением http://www. mathnet.ru/rus/agreement

Параметры загрузки:

IP : 54.224 .60 .19

26 апреля 2023 г., 14:42:23

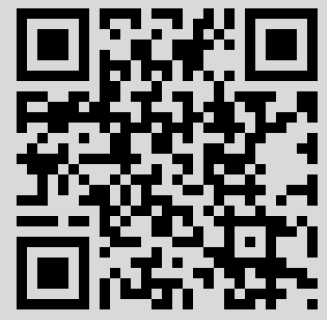




\title{
О РАЗЛОЖИМЫХ МНОГОЧЛЕННЫХ МАТРИЦАХ
}

\section{Б.3. Шаваровский}

\begin{abstract}
Доказьвается, что регулярная многочленная матрица над полем комплексных чисел с не более чем одним элементарным делителем степени 3 и всеми остальными элементарными делителями степени не вьше 2 допускает разложение на линейные регулярные множители.

Библиография: 14 названий.
\end{abstract}

1. Введение. В настоящей работе исследуется вопрос разложимости регулярнњх многочленных матриц (MМ) над полем комплексных чисел на линейные регулярные множители.

Вообще, задачам о структуре MМ, в частности их факторизации, посвящено много работ (см., например, монографии [1], [2] и цитированную там литературу). Одним из основных вопросов в построении теории разложения ММ есть поиск общего критерия выделяемости регулярного множителя и практическое построение разложения. Путем многократного применения разработанной методики к вновь получаемьм множителям ММ, в конце концов, можно, в принципе, прийти к далее неразложимым, в частности линейньг, множителям. Помимо того, возникает и другое направление: выделение классов ММ, разложимых на линейные множители, причем принадлежность к этим классам должна определяться по каким-то внешним признакам. Первьм из выделенных был класс $K_{1}$ ММ простой структуры [1], т.е. матрищ, все элементарные делители которых линейны (см. [3]-[5], а также [2, с. 113]). Казимирский и Петричкович анонсировали в [6] теорему, утверждающую разложимость на линейные регулярные множители квадратичных ММ, степени всех элементарных делителей которых не превьшают 2 . Далее, возможность разложения на линейные множители была доказана в [7], [8] [9] и [10] соответственно для класса $K_{2} \mathrm{MM}$, все характеристические корни которых имеют кратности, не превышающие 2 ; для класса $K_{3} \mathrm{MM}$, все коэффициенты которых являются попарно перестановочными матрицами простой структуры; для класса $K_{4}$ так назьваемых ММ квазипростой структуры (определение см. в п. 2) и для класса $K_{5} \mathrm{MM}$ степени $s$ порядка $n$, количество элементарных делителей которых не меньше $s(n-1)$. Наконец, Крупник в [11] (см. также [12]), используя результаты работы [7], доказал в общем случае теорему, сформулированную ранее Казимирским и Петричковичем для квадратичных ММ. Этим выделяется еще один класс $K_{6}$ разложимых ММ со всеми элементарными делителями степени не вьше 2. Имеет место следующее размещение 
найденньх классов: $\left(K_{1} \cup K_{2}\right) \subset K_{4} \subset K_{6}, K_{1} \subset K_{5}, K_{2} \cap K_{5}=K_{0} \neq \varnothing$. В $K_{0}$ содержится класс $K^{\prime}$ ММ без кратных характеристических корней, причем $K_{3} \cap K^{\prime} \neq \varnothing$.

В настоящей статье установлена разложимость на линейные регулярные множители регулярньх ММ с не более чем одним элементарным делителем степени 3 и со всеми остальными элементарными делителями степени не выше 2. Отмечается, что полученный результат в определенном смысле неулучшаемый. Его можно применить к исследованию разрешимости матричных многочленных односторонних уравнений (численные методы решения матричных уравнений см. в известной книге [13]).

2. Вспомогательные предложения. Будем рассматривать ММ над полем комплексных чисел $\mathbb{C}$. Согласимся с некоторыми определениями.

ММ назьвается простой по характеристическому корню $\alpha$, если все ее элементарные делители, соответствующие этому корню, линейны.

Очевидно, что простая по каждому своему характеристическому корню ММ является матрицей простой структуры.

ММ назьвается матрицей квазипростой структуры, если каждьй ее характеристический корень, по которому она не является простой, имеет кратность 2.

$\mathrm{MM} A(x)=A_{0} x^{s}+A_{1} x^{s-1}+\cdots+A_{s}$ назьвают регулярной, если $\operatorname{det} A_{0} \neq 0$.

Пусть $A(x)$ - регулярная ММ порядка $n$ степени $s$. Равенство

$$
S_{1} A(x)=\left(E_{n-m} \oplus B_{1}(x)\right) A_{1}(x)
$$

где $S_{1} \in \mathrm{GL}(n, \mathbb{C}), B_{1}(x)$ - регулярная $(m \times m)$-матрица степени $d, m \geqslant 1,1 \leqslant d<s$, $E_{n-m}$ - единичная матрица порядка $n-m$, называют разложсением MM $A(x)$. Если, в частности, $m=n$, то говорят, что из ММ $A(x)$ выделяется множитель степени $d$.

Пусть дано разложение (1) и разложение

$$
S_{2} A(x)=\left[E_{n-m-p} \oplus\left\|\begin{array}{cc}
B_{2}(x) & * \\
0 & B_{1}(x)
\end{array}\right\|\right] A_{2}(x),
$$

где $B_{1}(x)$ и $B_{2}(x)$ - матрицы порядков $m$ и $p$ соответственно, $p>0$. Тогда говорят, что разложсние (1) можно продолжить до разложсения (2). Разложение (2) назьвается максимальным продолжсением разложения (1), если число $p$ в этом разложении максимальное.

Пусть наряду с (1) существует разложение

$$
S_{3} A(x)=\left(E_{n-l} \oplus B_{3}(x)\right) A_{3}(x)
$$

где $B_{3}(x)-(l \times l)$-матрица, $\operatorname{deg} B_{3}(x)=\operatorname{deg} B_{1}(x)$ и $l>m$. Тогда разложение $(3)((1))$ назьвают бо́льшим (меньиим) разложением, чем разложение (1) ((3)).

В дальшейшем через $d^{C}(x)$ будем обозначать НОД миноров максимального порядка произвольной матрицы $C(x)$. Запись $H^{(i, j)}(x)$ будет означать матрицу, полученную из произвольной матрицы $H(x)$ вычеркиванием первых $i$ строк и первых $j$ столбцов. Через $t$ и $T$ обозначим операции транспонирования и блочного транспонирования соответственно. 
ЛЕмма 1. Пусть имеется разлохение

$$
S A(x)=\left(E_{n-m} \oplus\left(E_{m} x-B\right)\right) G(x),
$$

где $G(x)=\left\|A_{0}(x) \quad C(x)\right\|^{T}$, блок $C(x)$ состоит из $m$ строк. Eсли существует корень $\alpha$ многочлена $f(x)=(\operatorname{det} G(x)) / d^{C}(x)$ такой, что $\operatorname{def} G(\alpha)>\operatorname{def} C(\alpha)(\operatorname{def}-$ дефект), то разложение (4) можно продолжить до разложения

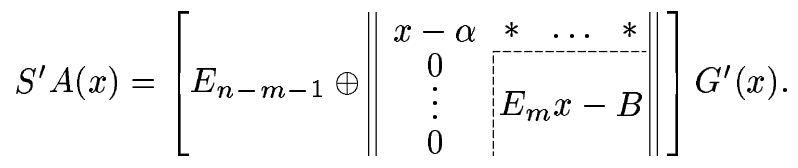

ДокаЗАтЕЛЬСтво. Пусть $\operatorname{def} G(\alpha)=p, \operatorname{def} C(\alpha)=q$. Тогда в матрище $C(\alpha)$ существует $m-q$ линейно независимых строк. Пусть без ограничения общности такими будут последние $m-q$. Так как $\operatorname{rank} G(\alpha)=n-p$ и $p>q$, то $n-q>n-p$. Следовательно, система, состоящая из $n-m$ строк матрицы $A_{0}(\alpha)$ и $m-q$ последних строк матрицы $C(\alpha)$, линейно зависима, причем ее подсистема из $m-q$ последних строк матрицы $C(\alpha)$ линейно независима. Это значит, что существует $S^{\prime} \in \operatorname{GL}(n, \mathbb{C})$ такая, что имеет место разложение (5).

\section{Из леммы 1 вытекает}

ЛЕмма 2. Пусть имеется разложсение (4) и многочлен $f(x)$, определенные условиями леммы 1. Если существует корень а многочлена $f(x)$, по которому матрица $G(x)$ проста, то разложение (4) мохно продолжить до разлохсения (5). Если, в частности, матрииа $G(x)$ является простой по каждому корню многочлена $f(x)$, за исключением, возмохкно, одного корня, кратность которого в многочлене $\operatorname{det} G(x)$ равна 2 , то из $\mathrm{MM} A(x)$ выделяется линейный множитель.

Лемма 3. Регулярная ММ $A(x)$ порядка $n$, каждый характеристический корень $\alpha$ которой удовлетворяет одному из следующих условий:

а) все әлементарные делители, соответствующие корню $\alpha$, линейны;

б) кратность корня а равна 2;

в) не более чем для одного 3-кратного корня $\alpha$ имеем $\operatorname{rank} A(\alpha)=n-1$, допускает выделение линейного множителя.

ДокАЗАтЕЛЬСтво. Напомним [1], что значением произвольной MМ $V(x)$ на системе корней многочлена $\varphi(x)$, заданного своим каноническим разложением $\varphi(x)=$ $\left(x-\alpha_{1}\right)^{s_{1}} \cdots\left(x-\alpha_{l}\right)^{s_{l}}$, назьвается числовая матрица

$$
M_{V(x)}(\varphi)=M_{V(x)}\left[\alpha_{1}^{\left(s_{1}\right)}, \ldots, \alpha_{l}^{\left(s_{l}\right)}\right]=\left\|W_{1} \ldots W_{l}\right\|^{T}
$$

где $W_{i}=\left\|V\left(\alpha_{i}\right) V^{(1)}\left(\alpha_{i}\right) \ldots V^{\left(s_{i}-1\right)}\left(\alpha_{i}\right)\right\|^{T}(i=1, \ldots, l), V^{(h)}(x)-h$-я производная матрицы $V(x)$. Значением ММ $V(x)$, состоящей из строк $\bar{v}_{1}(x), \ldots, \bar{v}_{n}(x)$, на системе корней әлементов матрицы $D(x)=\operatorname{diag}\left(d_{1}(x), \ldots, d_{n}(x)\right)$ называют [14] матрицу $M_{V(x)}(D)=\left\|M_{\bar{v}_{1}(x)}\left(d_{1}\right) \ldots M_{\bar{v}_{n}(x)}\left(d_{n}\right)\right\|^{T}$, где $M_{\bar{v}_{i}(x)}\left(d_{i}\right)$ отсутствует, если 
$d_{i}(x)=$ const. Далее через $U_{g}$ будем обозначать множество корней произвольного многочлена $g(x) \in \mathbb{C}[x]$, а через $A_{*}(x)$ - матрицу, взаимную с матрицей $A(x)$.

Если MМ $A(x)$ имеет простую структуру, то доказательство леммы 3 следует из леммы 2.

Пусть $\alpha_{1}, \ldots, \alpha_{u}$ - все (попарно различные) корни последнего инвариантного множителя $\varphi_{n}(x) \mathrm{MM} A(x)$ и пусть $\varphi(x)=\varphi_{n}(x) /\left(\left(x-\alpha_{1}\right) \cdots\left(x-\alpha_{u}\right)\right), k=\operatorname{deg} \varphi$, $m=\operatorname{rank} M_{A_{*}(x)}(\varphi)$. Далее возможны два случая:

1) $m=\min (k, n)$;

2) $m<\min (k, n)$.

Рассмотрим случай 1$)$. Если $k \geqslant n$, то для некоторого делителя $\psi(x)$ степени $n$ многочлена $\varphi(x)$ имеем $\operatorname{rank} M_{A_{*}(x)}(\psi)=n$, что согласно теореме 2 из [1, разд. II, §3] доказывает нашу лемму. Если $k<n$, то согласно теореме 1 из [1, разд. II, § 3] сушествует разложение $R A(x)=\left(E_{n-k} \oplus\left(E_{k} x-B\right)\right) D(x)$, где $\operatorname{det}\left(E_{k} x-B\right)=\varphi(x)$. Как легко видеть, к этому разложению можно применить лемму 2 и, таким образом, получить доказательство леммы 3.

Покажем, что в случае 2$)$ из MM $A(x)$ выделяется линейный множитель. Доказательство этого проведем от противного.

По теореме 4 из [1, разд. II, §4] существует разложение

$$
R A(x)=\left(E_{n-m} \oplus\left(E_{m} x-B\right)\right)\|*: C(x)\|^{T}, \quad m<n,
$$

где

$$
\operatorname{det}\left(E_{m} x-B\right)|\varphi(x)| \operatorname{det}\left(E_{m} x-B\right) d^{C}(x)
$$

(| - делит). Каждому разложению вида (6) с условиями (7) соответствует некоторое максимальное продолжение. Из всех таких продолжений выбираем наибольшее. Пусть это будет разложение

$$
R_{0} A(x)=\left\|\begin{array}{|l|l|l}
E_{n-m-p} & & \\
\hdashline & E_{p} x-B_{1} & B_{0} \\
\hdashline & & E_{m} x-B
\end{array}\right\| \cdot\left\|\begin{array}{l}
A_{0}(x) \\
\hdashline F(x) \\
\hdashline C(x)
\end{array}\right\| \quad(m+p<n) .
$$

Обозначим через $\beta$ тот характеристический 3 -кратный корень $\mathrm{MM} A(x)$, для которого $\operatorname{rank} A(\beta)=n-1$ (если такой существует). И пусть $\nu_{1}$ и $\nu_{2}-$ кратности этого корня в характеристическом многочлене левого сомножителя разложения (8) и в многочлене $d^{C}(x)$ соответственно. Далее возможны следующие случаи:
a) $\nu_{1}=3, \nu_{2}=0$
b) $\nu_{1}=2, \nu_{2}=1$;
c) $\nu_{1}=1, \nu_{2}=1$ (или $\left.\nu_{2}=2\right)$;
d) $\nu_{1}=0, \nu_{2}=2$ (или $\left.\nu_{2}=3\right)$;
е) $\nu_{1}=0, \nu_{2}=0$ (т.е. такой корень $\beta$ в многочлене $\operatorname{det} A(x)$ отсутствует). 
Случай, когда $\nu_{1}=2, \nu_{2}=0$, приводится, как легко убедиться, к случаю а). Мы можем предполагать, не ограничивая обшности, что в каждом из случаев а)-е) разложение (8) имеет один из следующих видов: случай а)

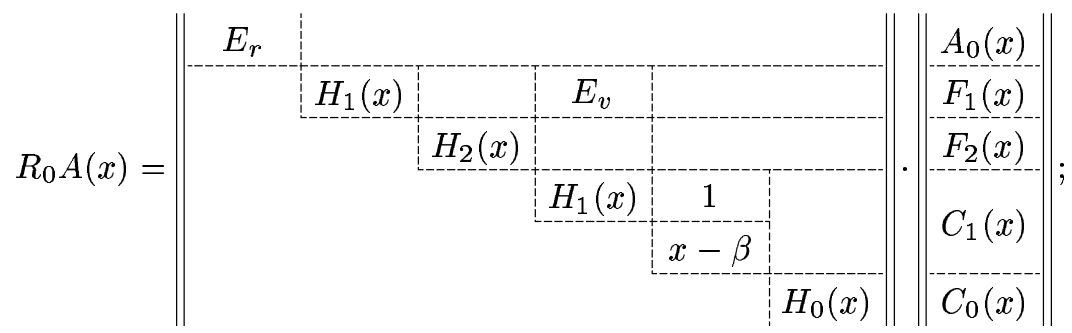

случай b)

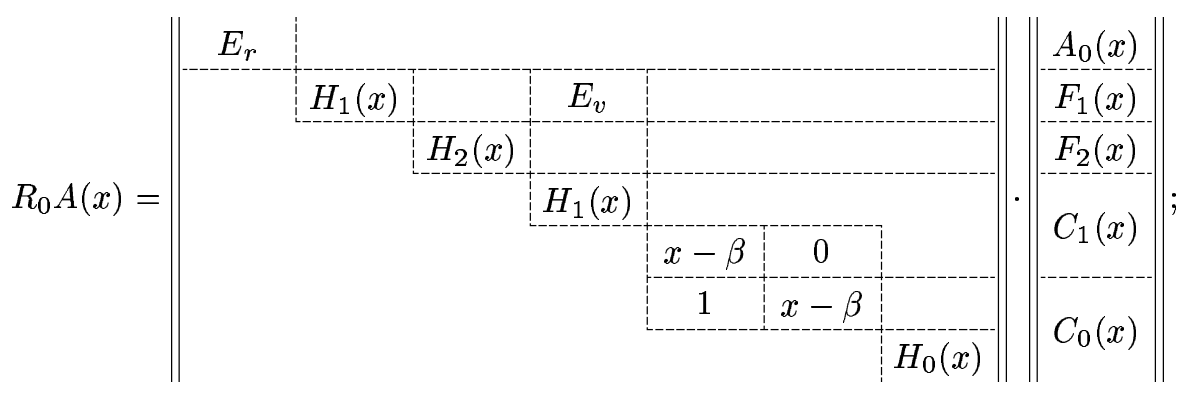

случай с)

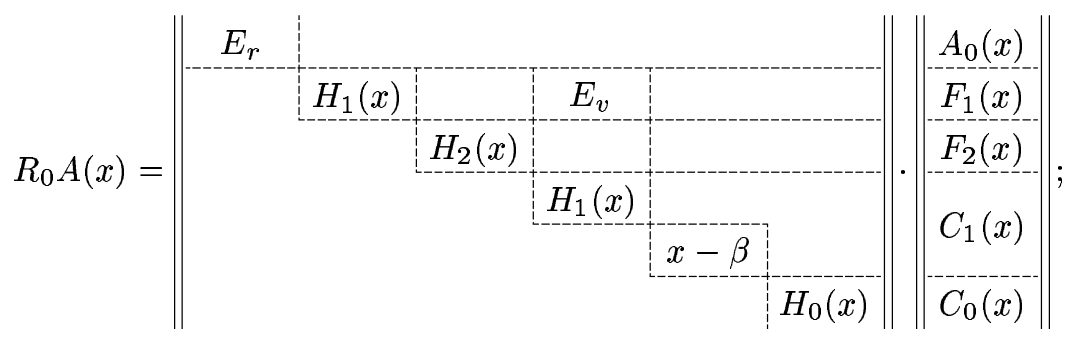

случаи d), e)

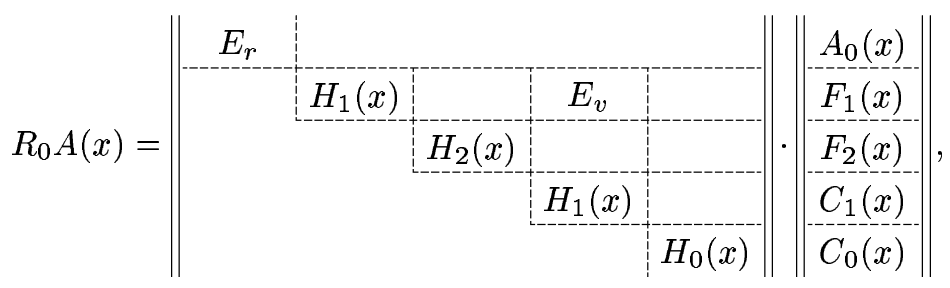

где $\left\|F_{1}(x) F_{2}(x)\right\|^{T}=F(x),\left\|C_{1}(x) C_{0}(x)\right\|^{T}=C(x)$ и блоки $H_{0}(x), H_{1}(x), H_{2}(x)$ диагональные, $r=n-m-p$. Заметим, что в случаях с) и d) $\beta \in U_{d^{C_{1}}}$ (иначе эти случаи приводятся соответственно к случаям b) и с)). 
Пусть $G_{0}(x)$ - второй сомножитель в разложениях $(9)-\left(9^{\prime \prime \prime}\right)$ и

$$
D_{0}(x)=\left\|F_{1}(x) F_{2}(x) C_{1}(x) C_{0}(x)\right\|^{T}
$$

Рассмотрим многочлен $f_{0}(x)=d^{G_{0}}(x) / d^{D_{0}}(x)$. Поскольку $f_{0}(x) \mid \varphi(x)$ и $f_{0}(x) \mid d^{C}(x)$ (иначе разложения (9)-( $\left.9^{\prime \prime \prime}\right)$ согласно лемме 2 можно продолжить, что невозможно), то все корни многочлена $f_{0}(x)$ просты и существует минимальная подматрица $Q_{0}(x)$ матрицы $C(x)$ (по строкам) такая, что $f_{0}(x) \mid d^{Q_{0}}(x)$. Покажем, что $Q_{0}(x)$ является подматрицей матрицы $C_{1}(x)$. В самом деле, если $C_{1}(x)=C(x)$, т.е. $C_{0}(x)$ - пустая матрица, то нечего доказывать. Если же $C_{0}(x)$ - непустая матрища и $Q_{0}(x)$ содержит, скажем, последнюю строку матрицы $C_{0}(x)$, то от разложения $(9)\left(\left(9^{\prime}\right)-\left(9^{\prime \prime \prime}\right)\right)$ можно перейти к разложению, отличающемуся от предыдущего только $n$-ми строками обоих сомножителей, причем $n$-й столбец первого сомножителя будет иметь вид $\|0 \ldots 0 x-\alpha\|^{t}, \mathrm{a}$ $n$-я строка второго - вид $\left(x-\gamma_{0}\right) \bar{a}(x)$, где $\gamma_{0} \in U_{f_{0}}$. Далее, от полученного разложения можно перейти к разложению, в котором $n$-й столбец первого сомножителя имеет вид $\left\|0 \ldots 0 x-\gamma_{0}\right\|^{t}$, а $n$-я строка второго - вид $(x-\alpha) \bar{a}(x)$. Но в таком разложении второй сомножитель будет простой матрицей по характеристическому корню $\gamma_{0}$, и согласно лемме 2 его можно продолжить, что противоречит максимальности разложения (8) с условиями (7).

Обозначим: $m_{0}=\operatorname{deg} f_{0}(x), l_{0}$ - число строк матрицы $Q_{0}(x), s=\operatorname{deg} A(x)$. Очевидно, что $m_{0} \geqslant s r(r=n-m-p)$ и, так как $m_{0} \leqslant(s-1) l_{0}$, то $l_{0} \geqslant s r /(s-1)$, поэтому $l_{0} \geqslant 2$. Не ограничивая общности, можем считать, что матрица $Q_{0}(x)$ содержит по крайней мере $l_{0}-1$ первых строк матрицы $C_{1}(x)$.

Фиксируем первые $l_{0}-1$ диагональных элементов $x-\alpha_{1}, \ldots, x-\alpha_{l_{0}-1}$ блока $H_{1}(x)$ (возможно, $\alpha_{i}=\beta$ для некоторого $\left.i<l_{0}\right)$ и от разложения $(9)\left(\left(9^{\prime}\right)-\left(9^{\prime \prime \prime}\right)\right)$ перейдем к меньшему разложению вида

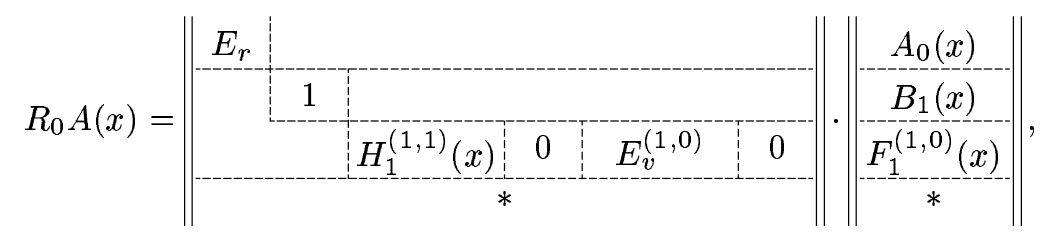

где $B_{1}(x)=\bar{b}_{1}(x)=\left(x-\alpha_{1}\right) \bar{u}_{1}(x)+\bar{c}_{1}(x), \bar{u}_{1}(x), \bar{c}_{1}(x)$ - первые строки матриц $F_{1}(x)$, $C_{1}(x)$ соответственно, символ “*” обозначает оставшиеся без изменений части матриц.

Рассмотрим теперь многочлен $f_{1}(x)=d^{G_{1}}(x) /\left(\left(x-\alpha_{1}\right) d^{D_{1}}(x)\right)$, где

$$
G_{1}(x)=\left\|\begin{array}{c}
\bar{b}_{1}(x) \\
D_{1}(x)
\end{array}\right\|, \quad D_{1}(x)=\left\|\begin{array}{c}
F_{1}^{(1,0)}(x) \\
F_{2}(x) \\
C(x)
\end{array}\right\|, \quad C(x)=\left\|\begin{array}{c}
C_{1}(x) \\
C_{2}(x)
\end{array}\right\|
$$

и покажем, что

$$
f_{1}(x)\left|\varphi(x), \quad f_{1}(x)\right| d^{C}(x)
$$


Если $\operatorname{deg} f_{1}(x)=0$, то все ясно. Если же $\operatorname{deg} f_{1}(x)>0, \gamma_{1} \in U_{f_{1}}$ и $\gamma_{1} \notin U_{\varphi}$ либо $\gamma_{1} \notin U_{d^{C}}$, то по лемме 2 от разложения (10) можно перейти к разложению

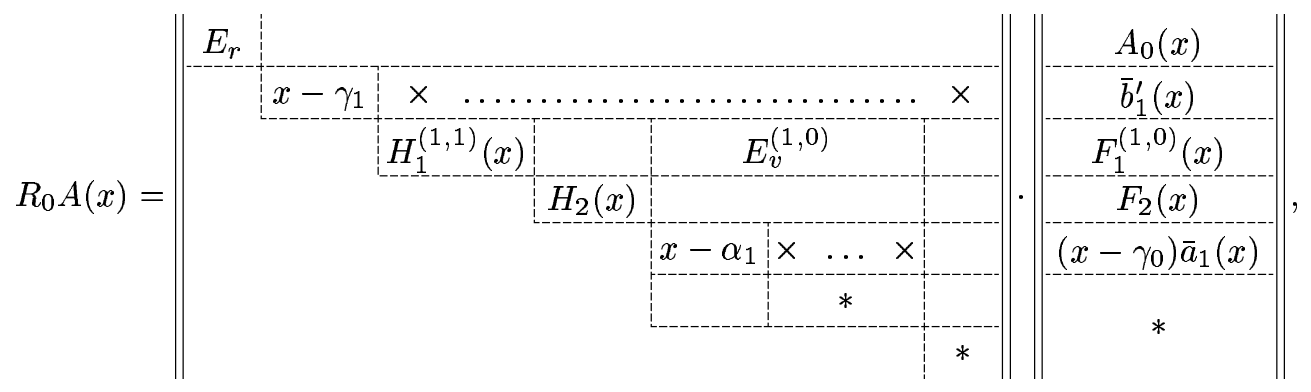

где $\gamma_{0} \in U_{f_{0}}$, символ “ $\times$ " означает некоторые элементы, а “*” - части матриц, не претерпевшие изменений. Так как $\gamma_{1} \neq \alpha_{0}$, то в левом сомножителе все недиагональные элементы столбца, содержащего элемент $x-\alpha_{1}$, можем считать нулевыми. Таким образом, в этом разложении можно поменять местами $(n-m+1)$-й диагональный элемент $x-\alpha_{1}$ первого сомножителя и множитель $x-\gamma_{0}$ из $(n-m+1)$-й строки второго сомножителя. Но тогда полученное разложение по лемме 2 можно продолжить, что противоречит максимальности разложения (8) с условиями (7). Следовательно, мы показали справедливость условий (11). Ясно также, что если $\operatorname{deg} f_{1}(x)>0$, то все корни многочлена $f_{1}(x)$ простые и НОД $\left(f_{0}(x), f_{1}(x)\right)=1$.

Так как $f_{1}(x) \mid d^{C}(x)$, то в матрице $C(x)$ существует минимальная подматрица $Q_{1}(x)$ (по строкам), которая вместе с ранее определенной подматрицей $Q_{0}(x)$ образуют матрицу $Q_{01}(x)=\left\|Q_{0}(x) Q_{1}(x)\right\|^{T}$ такую, что $f_{1}(x) \mid d^{Q_{01}}(x)$. Покажем, что матрица $Q_{1}(x)$ является подматрицей матрицы $C_{1}(x)$. Действительно, если $C_{1}(x)=C(x)$, т.е. $C_{0}(x)-$ пустая матрица, или $f_{1}(x) \mid d^{Q_{0}}(x)$, т.е. $Q_{1}(x)$ - пустая матрица, то уже все доказано. Если же матрица $Q_{1}(x)$ содержит, например, последнюю строку матрицы $C_{0}(x)$, то из разложения (10) имеем

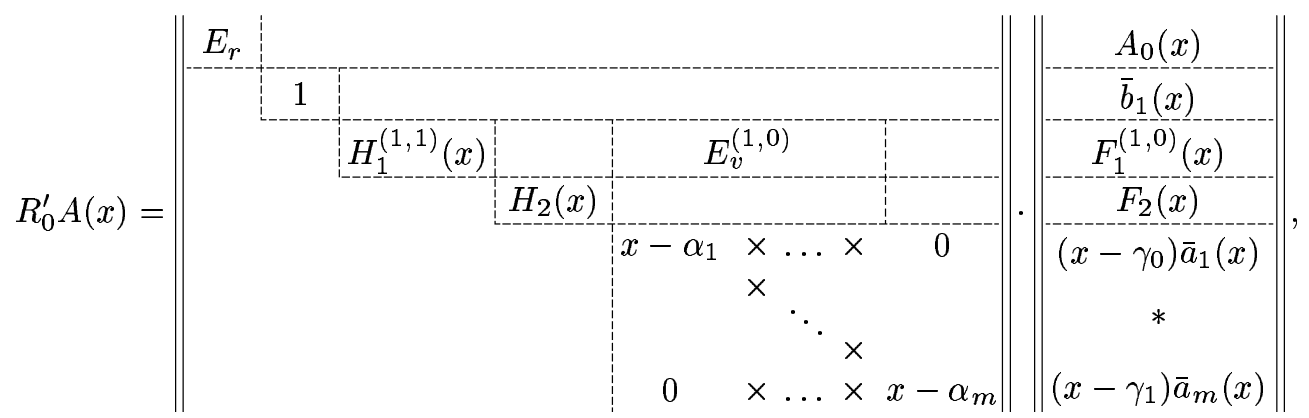

где $\gamma_{i} \in U_{f_{i}}, i=0,1$, символ "Х" означает некоторые элементы, а “*” - оставшиеся без изменений части матриц. В этом разложении $(n-m+1)$-й и $n$-й диагональные элементы $x-\alpha_{1}$ и $x-\alpha_{m}$ первого сомножителя можно поменять местами с множителями $x-\gamma_{0}$ и $x-\gamma_{1}$ соответствуюших строк второго сомножителя. В полученном разложении второй сомножитель будет простой матрицей по характеристическим корням $\gamma_{0}, \gamma_{1}$. Следовательно, это разложение можно последовательно дважды продолжить согласно 
лемме 2. Но тогда мы придем к разложению, большему, чем разложение (8), с условиями, аналогичными условиям (7), что невозможно.

Обозначим далее: $m_{i}=\operatorname{deg} f_{i}(x), d_{i}=\operatorname{deg} d^{D_{i}}(x), i=0,1$ ( $D_{0}(x)$ см. выше), $g_{1}=\operatorname{deg} d^{G_{1}}(x), l_{1}$ - число строк матрицы $Q_{01}(x)$. Так как $m_{1}=g_{1}-d_{1}-1, g_{1}=d_{0}+1$, то $m_{1}=d_{0}-d_{1}$, а поскольку $m_{0}=s n-m-p-d_{0}$ и $d_{1} \leqslant(s-1)(m+p-1)$, то $m_{0}+m_{1} \geqslant s(r+1)-1$. С другой стороны, $m_{0}+m_{1} \leqslant l_{1}(s-1)$. Таким образом, $l_{1} \geqslant(s(r+1)-1) /(s-1)$, поэтому $l_{1} \geqslant 3$. Допустим, не ограничивая общности, что матрица $Q_{01}(x)$ содержит не меньше, чем $l_{1}-1$ первых строк матрицы $C_{1}(x)$.

Фиксируем теперь $l_{1}-1$ первых диагональных элементов блока $H_{1}(x)$ в разложении $(9)\left(\left(9^{\prime}\right)-\left(9^{\prime \prime \prime}\right)\right)$. Пусть это будут элементы $x-\alpha_{1}, \ldots, x-\alpha_{l_{0}-1}, x-\alpha_{l_{0}}, \ldots, x-\alpha_{l_{1}-1}$.

Перейдя от разложения $(9)\left(\left(9^{\prime}\right)-\left(9^{\prime \prime \prime}\right)\right)$ к разложению $(10)$, предполагаем далее по индукции, что мы уже получили разложение

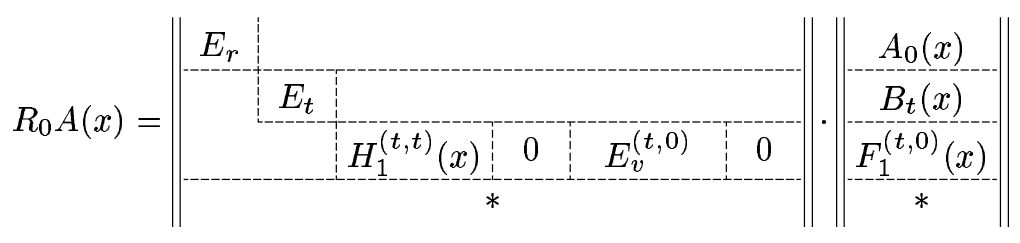

и попарно взаимно простые многочлены

$$
f_{0}(x)=\frac{d^{G_{0}}(x)}{d^{D_{0}}(x)}, \quad f_{i}(x)=\frac{d^{G_{i}}(x)}{\left(x-\alpha_{i}\right) d^{D_{i}}(x)}, \quad i=1, \ldots, t
$$

где $B_{t}(x)=\left\|\bar{b}_{1}(x) \ldots \bar{b}_{t}(x)\right\|^{T}, \bar{b}_{i}(x)=\left(x-\alpha_{i}\right) \bar{u}_{i}(x)+\bar{c}_{i}(x), \bar{u}_{i}(x), \bar{c}_{i}(x)-i$-е строки матриц $F_{1}(x), C_{1}(x)$ соответственно, символ “*” означает части матриц, не претерпевшие изменений,

$$
G_{i}(x)=\left\|\begin{array}{c}
\bar{b}_{i}(x) \\
D_{i}(x)
\end{array}\right\|, \quad D_{i}(x)=\left\|\begin{array}{c}
F_{1}^{(i, 0)}(x) \\
F_{2}(x) \\
C(x)
\end{array}\right\|, \quad C(x)=\left\|\begin{array}{c}
C_{1}(x) \\
C_{2}(x)
\end{array}\right\|,
$$

причем $f_{i}(x) \mid \varphi(x)$. Обозначив $m_{i}=\operatorname{deg} f_{i}(x), d_{i}=\operatorname{deg} d^{D_{i}}(x)(i=0,1, \ldots, t)$, по предположению индукции будем иметь $m_{1}+\cdots+m_{t}=d_{0}-d_{t}, m_{0}+m_{1}+\cdots+m_{t} \geqslant s(r+1)-i$. Кроме того, в матрице $C_{1}(x)$ выделены подматрицы $Q_{0}(x), Q_{1}(x), \ldots, Q_{t}(x)$ так, что в матрице $Q_{01 \ldots j}(x)=\left\|Q_{01 \ldots j-1}(x) Q_{j}(x)\right\|^{T}$ вьполняется условие $f_{j}(x) \mid d^{Q_{01 \ldots j}}(x)$ и никакая подматрища матрицы $Q_{01 \ldots j}(x)$ этим свойством не обладает $(j=0,1, \ldots, t)$. Матрица $Q_{01 \ldots j}(x)$ имеет $l_{j}$ строк. Некоторые из подматриц $Q_{i}(x)(i=1, \ldots, t)$, возможно, пустые. Тогда $l_{i}=l_{i-1}$, однако

$$
l_{i} \geqslant \frac{s(r+i)-i}{s-1}, \quad l_{i} \geqslant i+2, \quad i=1, \ldots, t
$$

Не ограничивая общности, предполагаем, что каждая из матриц $Q_{01 \ldots j}(x)(j=0,1$, $\ldots, t)$ содержит не меньше $l_{j}-1$ первых строк матрищы $C_{1}(x)$. 
$\Phi$ иксируем первые $l_{t}-1$ диагональных элементов $x-\alpha_{1}, \ldots, x-\alpha_{l_{t}-1}$ матрицы $H_{1}(x)$ (возможно, $\alpha_{i}=\beta$ для некоторого $i<l_{t}$ ). Поскольку согласно (13) имеем $t+1 \leqslant l_{t}-1$, то мы от разложения (12) можем перейти к разложению

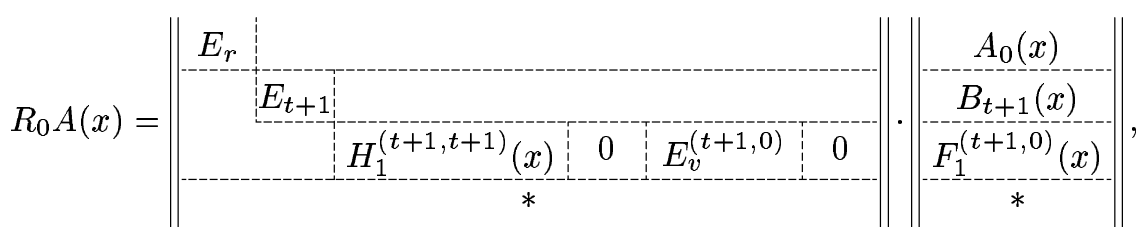

где $B_{t+1}(x)=\left\|B_{t}(x) \bar{b}_{t+1}\right\|^{T}, \bar{b}_{t+1}(x)=\left(x-\alpha_{t+1}\right) \bar{u}_{t+1}(x)+\bar{c}_{t+1}(x), \bar{u}_{t+1}(x), \bar{c}_{t+1}(x)-$ $(t+1)$-е строки матриц $F_{1}(x), C_{1}(x)$ соответственно.

Рассмотрим многочлен вида $f_{t+1}(x)=d^{G_{t+1}}(x) /\left(\left(x-\alpha_{t+1}\right) d^{D_{t+1}}(x)\right)$, где

$$
G_{t+1}(x)=\left\|\bar{b}_{t+1}(x)\right\|, \quad D_{t+1}(x)=\left\|\begin{array}{c}
F_{1}^{(t+1,0)}(x) \\
F_{2}(x) \\
C(x)
\end{array}\right\|, \quad C(x)=\left\|\begin{array}{c}
C_{1}(x) \\
C_{2}(x)
\end{array}\right\| .
$$

Очевидно, НОД $\left(f_{t+1}(x), \psi_{t}(x)\right)=1$, где $\psi_{t}(x)=\prod_{j=0}^{t} f_{j}(x)$. Покажем, что

$$
f_{t+1}(x)\left|\varphi(x), \quad f_{t+1}(x)\right| d^{C}(x) .
$$

Действительно, если $f_{t+1}(x)=$ const, то нечего доказьвать. Если же $\operatorname{deg} f_{t+1}(x)>0$ и существует корень $\gamma_{t+1} \in U_{f_{t+1}}$ такой, что $\gamma_{t+1} \notin U_{\varphi}$ либо $\gamma_{t+1} \notin U_{d^{C}}$, то согласно лемме 2 разложение (14) можно продолжить до разложения

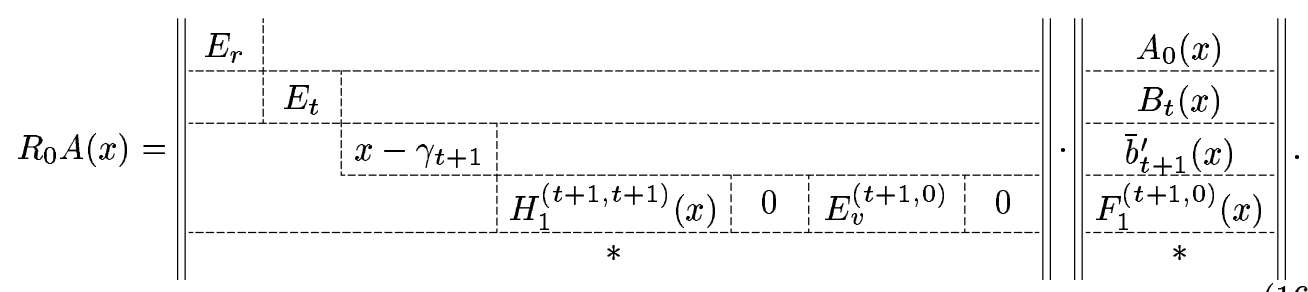

$\Phi$ иксируем $(t+1)$-й диагональный элемент $x-\alpha_{t+1}$ блока $H_{1}(x)$. Ему соответствует $(t+1)$-я строка блока $C_{1}(x)$. Поскольку $t+1 \leqslant l_{t}-1$ согласно $(13)$, то этой строке соответствует подматрища $Q_{t_{1}}$ матрищы $C_{1}(x)$, содержащая эту строку $\left(t_{1}<t\right)$. Подматрице $Q_{t_{1}}(x)$ соответствует многочлен $f_{t_{1}}(x) \not \equiv$ const. Фиксируем некоторый корень $\gamma_{t_{1}}$ многочлена $f_{t_{1}}(x)$ и $t_{1}$-й диагональный элемент $x-\alpha_{t_{1}}$ блока $H_{1}(x)$. Ему соответствует $t_{1}$-я строка блока $C_{1}(x)$. Поскольку $t_{1} \leqslant l_{t_{1}-1}-1$, то этой строке соответствует подматрица $Q_{t_{2}}(x)$ матрицы $C_{1}(x)$, содержащая эту строку $\left(t_{2}<t_{1}\right)$. Подматрище $Q_{t_{2}}(x)$ соответствует многочлен $f_{t_{2}}(x) \not \equiv$ const. Фиксируем некоторьй корень $\gamma_{t_{2}}$ многочлена $f_{t_{2}}(x)$ и $t_{2}$-й диагональньй элемент $x-\alpha_{t_{2}}$ блока $H_{1}(x)$ и т.д. Продолжая так и далее, мы, в конце концов, придем к подматрице $Q_{0}(x)$, многочлену $f_{0}(x) \not \equiv$ const и некоторому его корню $\gamma_{0}$. Таким образом, будут зафиксированы диагональные элементы $x-\alpha_{t+1}, x-\alpha_{t_{1}}, \ldots, x-\alpha_{t_{q}}\left(t+1>t_{1}>t_{2}>\cdots>t_{q}\right)$ в матрище $H_{1}(x)$ и корни $\gamma_{t_{1}}, \gamma_{t_{2}}, \ldots, \gamma_{0}$ многочленов $f_{t_{1}}(x), f_{t_{2}}(x), \ldots, f_{0}(x)$ соответственно. 
Теперь от (16) перейдем к разложению, которое отличается от предыдущего только тем, что на месте блоков $H_{1}(x), C_{1}(x)$ (в частях матриц, обозначенньх значком “*”) стоят соответственно блоки $\widetilde{H}_{1}(x), \widetilde{C}_{1}(x)$ вида

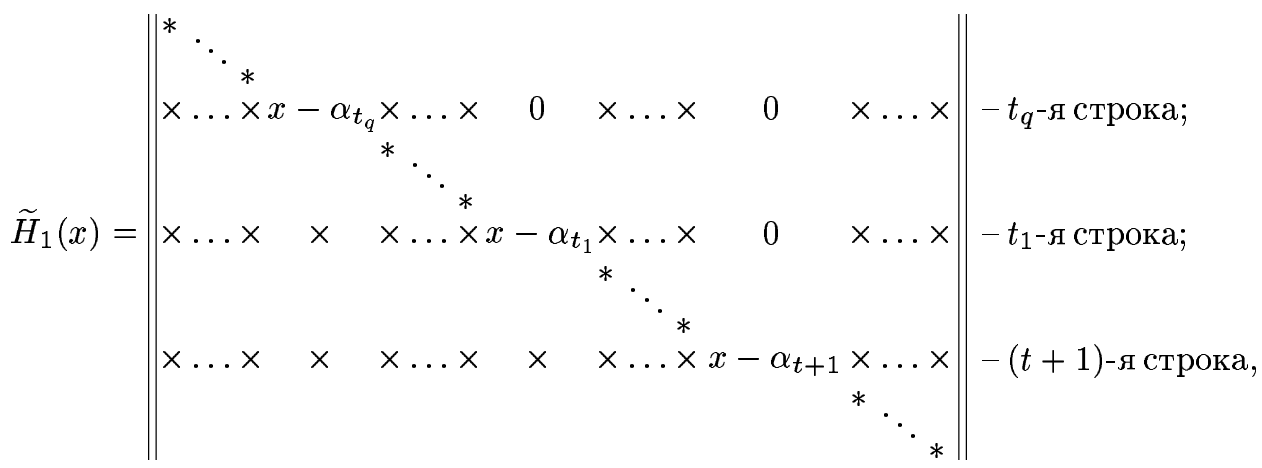

$$
\begin{aligned}
& \widetilde{C}_{1}(x)=\left\|\begin{array}{c}
* \\
\left(x-\gamma_{0}\right) \bar{a}_{t_{q}}(x) \\
* \\
\left(x-\gamma_{t_{2}}\right) \bar{a}_{t_{1}}(x) \\
* \\
\left(x-\gamma_{t_{1}}\right) \bar{a}_{t+1}(x) \\
*
\end{array}\right\|-t_{q^{-я} \text { строка }}
\end{aligned}
$$

где все необозначенные элементы равны нулю, значком " ”" обозначены некоторые элементы, а “*” - части матрищ, оставшиеся без изменений. Затем от полученного перейдем к разложению, в котором вместо блоков $\widetilde{H}_{1}(x), \widetilde{C}_{1}(x)$ стоят блоки $\widetilde{H}_{1}^{\prime}(x), \widetilde{C}_{1}^{\prime}(x)$, причем

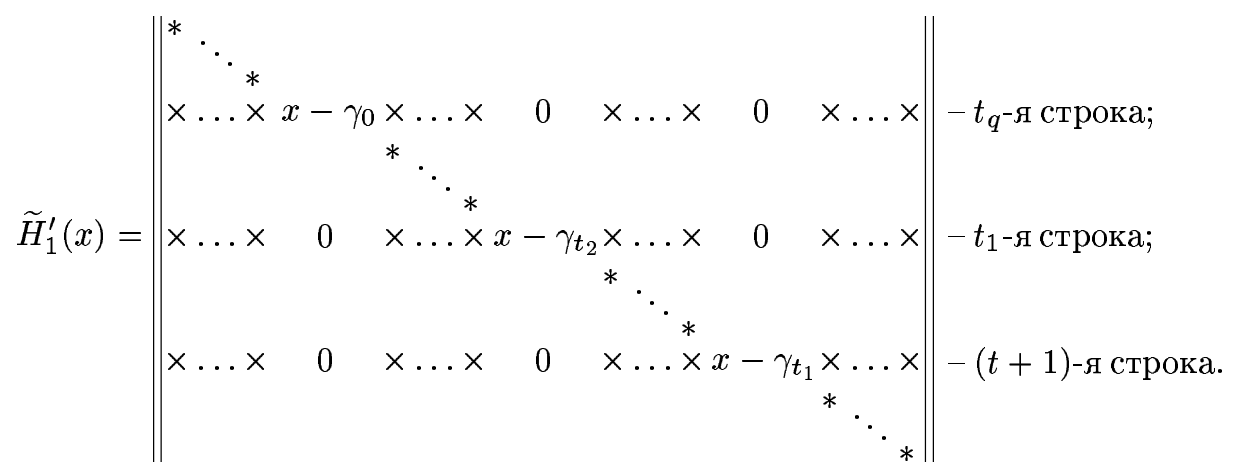

Пусть $x-\beta_{1}, x-\beta_{2}, \ldots, x-\beta_{t+1}-$ первые $t+1$ диагональных элементов блока $\widetilde{H}_{1}^{\prime}(x)$. Поскольку правьй сомножитель в полученном разложении будет простой матрицей по характеристическим корням $\beta_{1}, \beta_{2}, \ldots, \beta_{t+1}$, то согласно лемме 2 это разложение можно продолжить последовательно $t+1$ раз, что противоречит максимальности числа $p$ в разложении (8) с условиями (7).

Таким образом, мы показали, что многочлен $f_{t+1}(x)$ удовлетворяет условиям (15). 
Так как $f_{t+1}(x) \mid d^{C}(x)$, то существует минимальная подматрища $Q_{t+1}(x)$ матрицы $C(x)$ такая, что $f_{t+1}(x) \mid d^{Q_{01 \ldots t t+1}}(x)$, где $Q_{01 \ldots t t+1}(x)=\left\|Q_{01 \ldots t}(x) Q_{t+1}(x)\right\|^{T}$. Покажем, что $Q_{t+1}(x)$ является подматрицей матрицы $C_{1}(x)$. Вопреки этому допустим, что матрица $Q_{t+1}(x)$ содержит, скажем, последнюю строку матрицы $C_{0}(x)$. Тогда из разложения (14) можем получить разложение, которое отличается от него только блоками $H(x)=\operatorname{diag}\left\{H_{1}(x), H_{0}(x)\right\}, C(x)=\left\|C_{1}(x) C_{0}(x)\right\|^{T}$, а именно, на месте этих блоков стоят соответственно блоки $H^{\prime}(x), C^{\prime}(x)$ вида

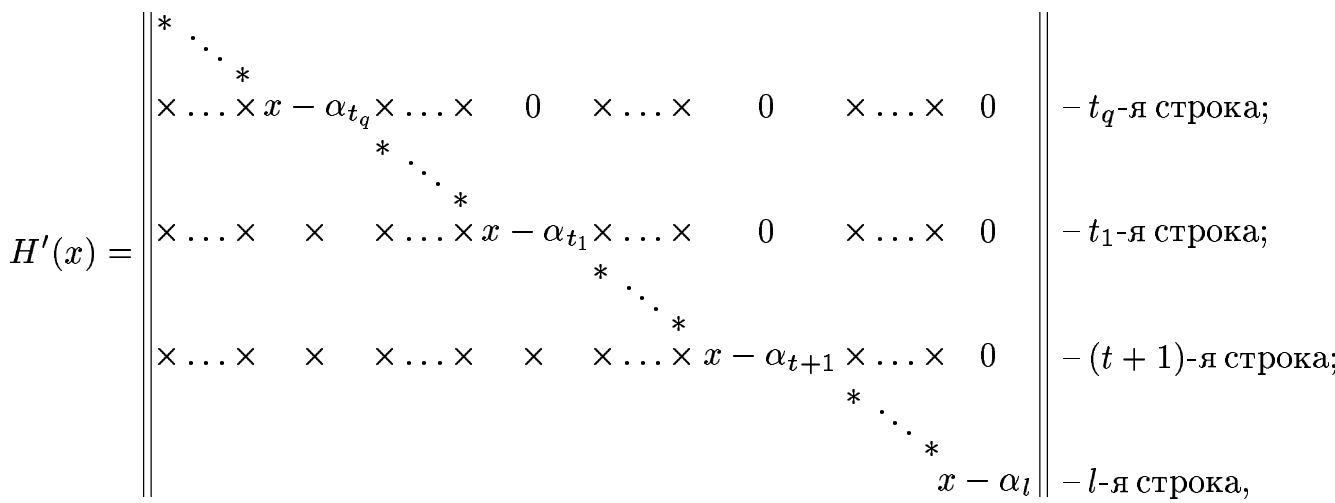

$$
\begin{aligned}
& C^{\prime}(x)=\left\|\begin{array}{c}
* \\
\left(x-\gamma_{0}\right) \bar{a}_{t_{q}}(x) \\
* \\
\left(x-\gamma_{t_{2}}\right) \bar{a}_{t_{1}}(x) \\
* \\
\left(x-\gamma_{t_{1}}\right) \bar{a}_{t+1}(x) \\
* \\
\left(x-\gamma_{t+1}\right) \bar{a}_{l}(x)
\end{array}\right\|-t_{q^{-} \text {-я строкок } ;}{ }_{-l \text {-я строка, }}
\end{aligned}
$$

где $\gamma_{t+1} \in U_{f_{t+1}}$, элементы $\alpha_{t_{q}}, \ldots, \alpha_{t_{1}}, \alpha_{t+1}, \gamma_{0}, \ldots, \gamma_{t_{2}}, \gamma_{t_{1}}$ определены как и раньше. Далее, от этого разложения перейдем к разложению, в котором вместо блоков $H^{\prime}(x), C^{\prime}(x)$ стоят соответственно блоки $H^{\prime \prime}(x), C^{\prime \prime}(x)$, причем

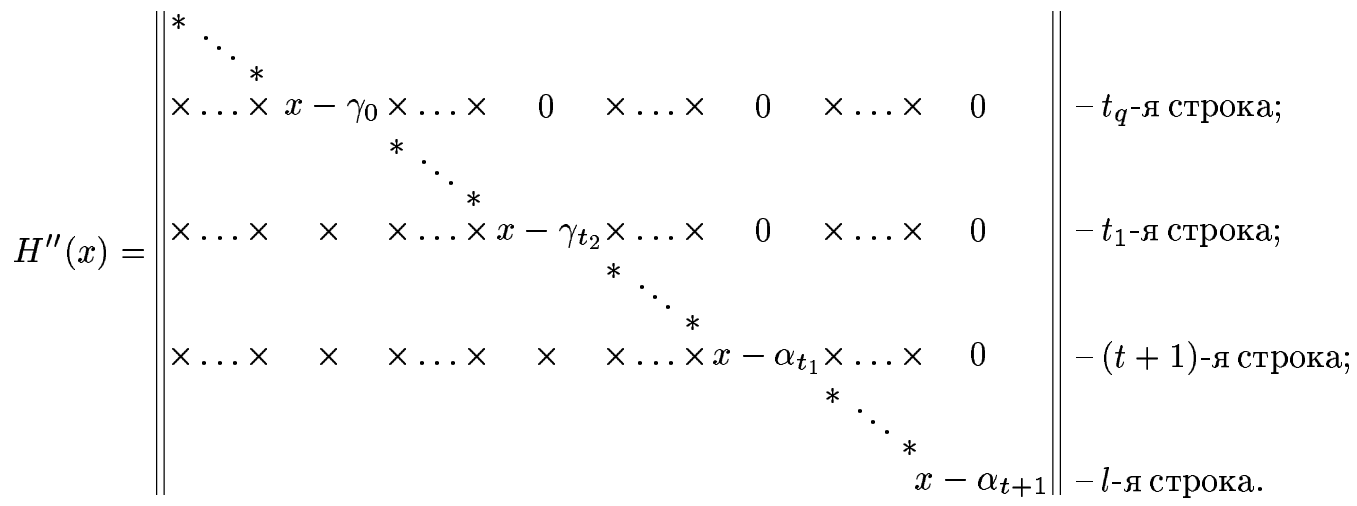


Обозначим диагональные элементы матрицы $H^{\prime \prime}(x)$ через $x-\beta_{1}, \ldots, x-\beta_{l}$. Поскольку в полученном разложении правый сомножитель будет простой матрицей по характеристическим корням $\beta_{l}, \beta_{t+1}, \ldots, \beta_{1}$, то это разложение можно продолжить последовательно $t+2$ раз, что невозможно.

Допустим без ограничения обшности, что подматрица $Q_{t+1}(x)$, состоящая из $l_{t+1}$ строк, содержит не менее $l_{t+1}-1$ первых строк матрицы $C_{1}(x)$. Обозначив $m_{t+1}=$ $\operatorname{deg} f_{t+1}(x), g_{t+1}=\operatorname{deg} d^{G_{t+1}}(x), d=\operatorname{deg} d^{D_{t+1}}(x)$, имеем $m_{t+1}=g_{t+1}-d_{t+1}-1$, $g_{t+1}=d_{t}+1, m_{t+1}=d_{t}-d_{t+1}$. В силу предположения индукции $m_{1}+\cdots+m_{t}=d_{0}-d_{t}$. Следовательно, $m_{1}+\cdots+m_{t}+m_{t+1}=d_{0}-d_{t+1}$ и так как $m_{0}=s n-m-p-d_{0}$, $d_{t+1} \leqslant(s-1)(m+p-t-1)$, то $m_{0}+m_{1}+\cdots+m_{t}+m_{t+1} \geqslant s(r+t+1)-t-1$. Но поскольку $m_{0}+m_{1}+\cdots+m_{t}+m_{t+1} \leqslant l_{t+1}(s-1)$, то $l_{t+1} \geqslant(s(r+t+1)-t-1) /(s-1)$, откуда $t+2 \leqslant l_{t+1}-1$.

Мы можем делать еще один шаг. Но, с другой стороны, мы, в конце концов, исчерпаем матрицу $C_{1}(x)$ и, скажем, уже $l_{t+2}$ должно стать бо́льшим, чем число ее строк, что невозможно. Полученное противоречие и доказьвает нашу лемму.

3. Основной результат. Он заключается в следующей теореме.

Теорема. Регулярная ММ $A(x)$ с не более чем одним әлементарным делителем степени 3 и со всеми остальными әлементарными делителями степени не выше 2 разложима в произведение линейных регулярных множителей.

ДокАЗАТЕЛЬСТво. Пусть $s$ и $n$-степень и порядок MМ $A(x)$ соответственно. Для доказательства достаточно показать выделяемость из MM $A(x)$ линейного регулярного множителя и применить индукцию по $s$. Мы с самого начала можем предполагать, что первьй инвариантньй множитель MM $A(x)$ равен 1 , иначе выделяемость нужного множителя очевидна. Согласно [1] матрица $A(x)$ полускалярно эквивалентньпи преобразованиями приводится к треугольному виду

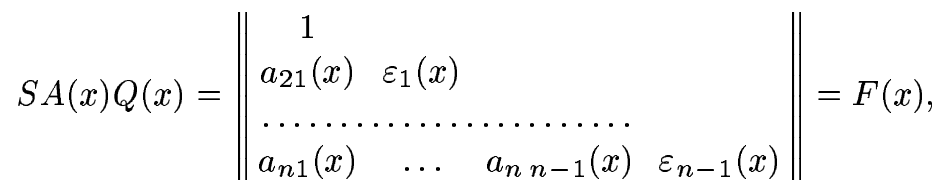

где $1, \varepsilon_{1}(x), \ldots, \varepsilon_{n-1}(x)$ - инвариантные множители. Стало быть, $\varepsilon_{i}(x)\left|\varepsilon_{i+1}(x), \varepsilon_{i}(x)\right|$ $a_{j i+1}(x)(j=i+2, \ldots, n, i=1, \ldots, n-2)$. Пусть $F_{*}(x)$ - матрица, взаимная с матрицей $F(x)$ :

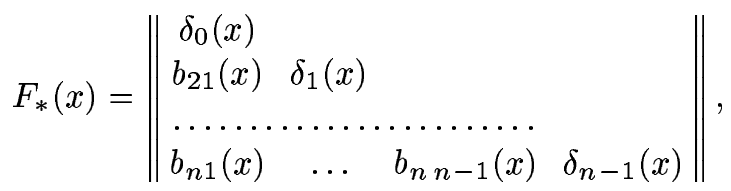

где $\delta_{0}(x)=\varepsilon_{1}(x) \cdots \varepsilon_{n-1}(x), \delta_{k}(x)=\delta_{0}(x) / \varepsilon_{k}(x)$. Очевидно, $\delta_{k}(x) \mid b_{k+1}(x)(k=1$, $\ldots, n-1, l=1, \ldots, k)$. Тогда $F_{*}(x)=\operatorname{diag}\left(\delta_{0}(x), \delta_{1}(x), \ldots, \delta_{n-1}(x)\right) P(x)$, где матрица $P(x)$ имеет вид

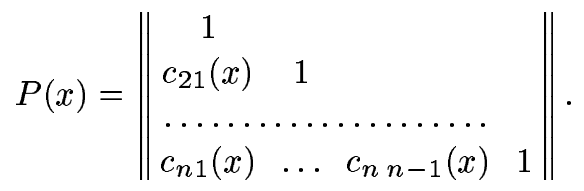


Поскольку $\operatorname{diag}\left(\delta_{0}(x), \delta_{1}(x), \ldots, \delta_{n-1}(x)\right) P(x) F(x)=\delta_{0}(x) E_{n}$, то, сократив (слева) последнее равенство на $\operatorname{diag}\left(\delta_{0}(x), \delta_{1}(x), \ldots, \delta_{n-1}(x)\right)$, находим

$$
P(x) F(x)=\operatorname{diag}\left(1, \varepsilon_{1}(x), \ldots, \varepsilon_{n-1}(x)\right) .
$$

Следовательно, произведение $P(x) S$, где $S$ взято из (17), является левой преобразующей матрицей $\mathrm{MM} A(x)$ к ее форме Смита $E(x)=\operatorname{diag}\left(1, \varepsilon_{1}(x), \ldots, \varepsilon_{n-1}(x)\right)$ $\left(\varepsilon_{i}(x) \mid \varepsilon_{i+1}(x)\right)$. Построим квадратную порядка sn числовую матрищу $P^{\prime}=$ $M_{P(x) \| E E x} E x^{s-1 \|}(E)$, которая состоит, очевидно, из горизонтальных полос вида

$$
\begin{aligned}
& \left\|\bar{a} \quad \alpha \bar{a} \quad \ldots \alpha^{s-1} \bar{a}\right\|, \\
& \left\|\begin{array}{cccc}
\bar{b} & \beta \bar{b} & \ldots & \beta^{s-1} \bar{b} \\
\bar{b}^{\prime} & \beta \bar{b}^{\prime}+\bar{b} & \ldots & \beta^{s-1} \bar{b}^{\prime}+(s-1) \beta^{s-2} \bar{b}
\end{array}\right\|, \\
& \left\|\begin{array}{cccc}
\bar{c} & \gamma \bar{c} & \ldots & \gamma^{s-1} \bar{c} \\
\bar{c}^{\prime} & \gamma \bar{c}^{\prime}+\bar{c} & \ldots & \gamma^{s-1} \bar{c}^{\prime}+(s-1) \gamma^{s-2} \bar{c} \\
\bar{c}^{\prime \prime} & \gamma \bar{c}^{\prime \prime}+2 \bar{c}^{\prime} & \ldots & \gamma^{s-1} \bar{c}^{\prime \prime}+2(s-1) \gamma^{s-2} \bar{c}^{\prime}+(s-1)(s-2) \gamma^{s-3} \bar{c}
\end{array}\right\|,
\end{aligned}
$$

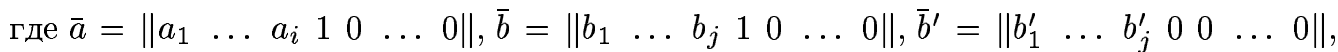
$\bar{c}=\left\|c_{1} \ldots c_{n-1} 1\right\|, \bar{c}^{\prime}=\left\|c_{1}^{\prime} \ldots c_{n-1}^{\prime} 0\right\|, \bar{c}^{\prime \prime}=\left\|c_{1}^{\prime \prime} \ldots c_{n-1}^{\prime \prime} 0\right\|, 1 \leqslant i, j \leqslant n-1$, $\alpha, \beta, \gamma$ - некоторые (возможно, равные) характеристические корни ММ $A(x)$, причем полоса ширины 3 единственная. Умножением (слева) каждой из полос матрицы $P^{\prime}$ соответственно на матрицы вида

$$
\|q\|, \quad\left\|\begin{array}{cc}
q & 0 \\
r & q
\end{array}\right\|, \quad\left\|\begin{array}{ccc}
q & 0 & 0 \\
r_{1} & q & 0 \\
r_{2} & r_{3} & q
\end{array}\right\| \quad(q \neq 0)
$$

и умножением справа матрицы $P^{\prime}$ на матрицу $\operatorname{diag}\{\underbrace{D, \ldots, D}_{s}\}$, где

$$
D=\left\|\begin{array}{ccccc}
1 & & & & 0 \\
& 1 & & & q_{1} \\
& & \ddots & & \vdots \\
& & & 1 & q_{n-2} \\
& & & & 1
\end{array}\right\|
$$

можно прийти к матрице $G$, в которой вместо полос вида (19)-(21) будут стоять соответственно полосы вида

$$
\begin{aligned}
& \left\|\bar{d} \alpha \bar{d} \ldots \alpha^{s-1} \bar{d}\right\|, \\
& \left\|\begin{array}{cccc}
\bar{f} & \beta \bar{f} & \ldots & \beta^{s-1} \bar{f} \\
\overline{f^{\prime}} & \beta \bar{f}^{\prime}+\bar{f} & \ldots & \beta^{s-1} \bar{f}^{\prime}+(s-1) \beta^{s-2} \bar{f}
\end{array}\right\|, \\
& \begin{array}{||cccc}
\bar{g} & \gamma \bar{g} & \ldots & \gamma^{s-1} \bar{g} \\
\bar{g}^{\prime} & \gamma \bar{g}^{\prime}+\bar{g} & \ldots & \gamma^{s-1} \bar{g}^{\prime}+(s-1) \gamma^{s-2} \bar{g} \\
\bar{g}^{\prime \prime} & \gamma \bar{g}^{\prime \prime}+2 \bar{g}^{\prime} & \ldots & \gamma^{s-1} \bar{g}^{\prime \prime}+2(s-1) \gamma^{s-2} \bar{g}^{\prime}+(s-1)(s-2) \gamma^{s-3} \bar{g}
\end{array} \|,
\end{aligned}
$$


где

$$
\begin{aligned}
& \bar{d}=\left\|d_{1} \ldots d_{n-1} 1\right\|, \quad \bar{f}=\left\|f_{1} \ldots f_{n-1} 1\right\|, \quad \bar{f}^{\prime}=\left\|f_{1}^{\prime} \ldots f_{n-1}^{\prime} 0\right\|, \\
& \bar{g}=\left\|g_{1} \ldots g_{n-1} 1\right\|, \quad \bar{g}^{\prime}=\left\|g_{1}^{\prime} \ldots g_{n-1}^{\prime} 0\right\|, \quad \bar{g}^{\prime \prime}=\left\|g_{1}^{\prime \prime} \ldots g_{n-1}^{\prime \prime} 0\right\| \text {. }
\end{aligned}
$$

Количество $p$ полос матрицы $G$ (так же, как и матрицы $P^{\prime}$ ) равно числу элементарных делителей $\mathrm{MM} A(x)$. Каждой полосе соответствует некоторьй характеристический корень MM $A(x)$. Различным полосам могут соответствовать одинаковые корни. Заменим теперь фигурирующий в каждой полосе характеристический корень некоторой переменной $x_{i}$ так, чтобы различные полосы “получили" различные переменные. Таким образом, мы придем к матрище $U\left(x_{1}, \ldots, x_{p}\right)$ над кольцом $\mathbb{C}\left[x_{1}, \ldots, x_{p}\right]$. Поскольку согласно лемме 1 из [14] $\operatorname{det} P^{\prime} \neq 0$, то $\operatorname{det} U\left(x_{1}, \ldots, x_{p}\right) \not \equiv 0$. Следовательно, можно найти попарно различные элементы $\alpha_{1}, \ldots, \alpha_{p} \in \mathbb{C}$ такие, что матрица $V$, полученная из матрищы $U\left(x_{1}, \ldots, x_{p}\right)$ подстановкой $x_{i}=\alpha_{i}(i=1, \ldots, p)$, неособенная. Каждому из зафиксированных элементов $\alpha_{i}(i=1, \ldots, p)$ припишем число $k_{i}$, равное ширине соответствующей ему полосы. Очевидно, $k_{1}, \ldots, k_{p}$ - степени всех элементарных делителей MM $A(x)$, поэтому $\sum k_{i}=s n$. Далее, по $m$-у столбцу $v_{m}$ матрицы $V$ найдем единственный элемент $h_{m}(x) \in \mathbb{C}[x], \operatorname{deg} h_{m}(x)<s n$ такой, что $M_{h_{m}(x)}\left[\alpha_{1}^{\left(k_{1}\right)}, \ldots, \alpha_{p}^{\left(k_{p}\right)}\right]=-v_{m}$ $(m=1, \ldots, n-1)$, и построим матрицу

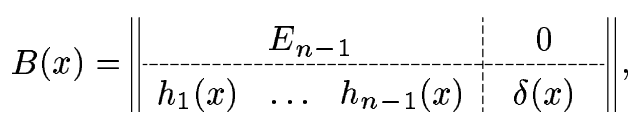

где $\delta(x)=\left(x-\alpha_{1}\right)^{k_{1}} \cdots\left(x-\alpha_{p}\right)^{k_{p}}$. Легко убедиться, что матрица

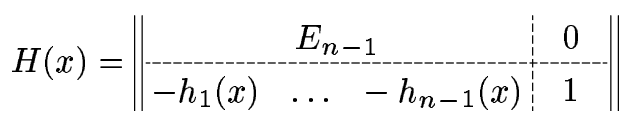

является левой преобразующей матрицей ММ $B(x)$ к ее форме Смита $\Delta(x)=\operatorname{diag}(1$, $\ldots, 1, \delta(x))$ и $M_{H(x) \| E} E x E x^{s-1} \|(\Delta)=V$. На основании упоминавшейся уже леммы 1 из [14] существует матрица $R(x) \in \mathrm{GL}(n, \mathbb{C}[x])$ такая, что $C(x)=B(x) R(x)$ - регулярная ММ. Согласно лемме 3 из MM $C(x)$ выделяется линейньй регулярньй множитель. Это значит, что на основании теоремы 5 и утверждения 4 из $[1$, разд. II, $\S \S 2,4]$ для некоторого делителя $\rho(x)$ степени $n$ многочлена $\delta(x)(n \times n)$-матрица $M_{\left\|h_{1}(x) \ldots h_{n-1}(x) 1\right\|}(\rho)$, являющаяся подматрищей $(s n \times n)$-матрищы $M_{\left\|h_{1}(x) \ldots h_{n-1}(x) 1\right\|}(\delta)$ полного ранга, неособенная. Следовательно, в $(s n \times n)$-матрище $M_{P(x)}(E)$ существует неособенная подматрица $M_{P(x)}(\Phi)$ порядка $n$, где $\Phi(x)$ - некоторый диагональный делитель ММ $E(x)$, т.е. $E(x)=\Phi(x) \Psi(x)$, причем $\operatorname{deg} \operatorname{det} \Phi(x)=n$. На основании (17) и (18) запишем равенство

$$
C A(x)=P^{-1}(x) \Phi(x) \Psi(x) Q^{-1}(x) .
$$

Обозначим $\varphi(x)=\operatorname{det} \Phi(x)$. Учитьвая неособенность матрицы $M_{P(x)}(\Phi)$ и структуру матрицы $M_{\Phi_{*}(x) P(x)}(\varphi)$, делаем вьвод, что последняя имеет ранг $n$. А это на основании теоремы 3 из [1, разд. III, §2] значит, что матрица $P^{-1}(x) \Phi(x)$ в соотношении (22) регуляризуется умножением справа на некоторую обратимую ММ. Поэтому $A(x)=M(x) N(x)$, где $M(x)$ - регулярная ММ степени 1. Теорема доказана. 
В заключение отметим, что полученньй результат нельзя улучшить, допуская наличие в ММ больше одного элементарного делителя степени 3 или же допуская наличие элементарного делителя степени 4.

Рассмотрим матричные уравнения вида

$$
\begin{aligned}
& X^{s}+X^{s-1} A_{1}+\cdots+X A_{s-1}+A_{s}=0 \\
& X^{s}+A_{1} X^{s-1}+\cdots+A_{s-1} X+A_{s}=0
\end{aligned}
$$

где $X$ - неизвестная $(n \times n)$-матрица, и соответствующую им ММ

$$
A(x)=E_{n} x^{s}+A_{1} x^{s-1}+\cdots+A_{s-1} x+A_{s} .
$$

СлЕдСТВИЕ. Если ММ $A(x)$ удовлетворяет условиям теоремы, то каждое из уравнений (23) разрешимо.

\section{СПИСОК ЦИТИРОВАННОЙ ЛИТЕРАТУРЫ}

[1] Казімірський П. С. Розклад матричних многочленів на множники. Київ: Наукова думка, 1981.

[2] Gochberg I., Lancaster P., Rodman L. Matrix Polynomials. New York: Acad. Press, 1982.

[3] Маркус А. С., Мереуца И.В. О некоторых свойствах простых $\lambda$-матриц // Матем. исслед. 1975. Т. 10. № 3. С. 207-213.

[4] Казимирский П. С. Выделение из матричного многочлена регулярного линейного множителя простой структуры // Теоретические и прикладные вопросы алгебры и дифференциальных уравнений. Киев: Институт математики АН УССР, 1976. С. 29-40.

[5] Сахнович Л.А. О факторизации передаточных оператор-функций // Докл. АН СССР. 1976. T. 226. № 4. C. 781-784.

[6] Казимирский П. С., Петричкович В.М. Одно достаточное условие разложимости матричного квадратного трехчлена на линейные множители // II Всесоюзный симпозиум по теории колец, алгебр и модулей. Резюме сообщений. Кишинев: Штиинца, 1974. С. 29-30.

[7] Казимирский П. С., Петричкович В.М. Разложимость полиномиальных матриц на линейные множители // Матем. методы и физ. -мех. поля. 1978. № 8. С. 3-9.

[8] Зелиско В. Р., Шаваровский Б. З. Разложение матричного многочлена в произведение множителей простой структуры // Матем. методы и физ. -мех. поля. 1982. № 15. С. 43-48.

[9] Шаваровский Б. З. Преобразования подобия матричных многочленов и их разложимость на множители. Дисс. . . к. ф.-м.н. Львов: ИППММ, 1985.

[10] Мельник О.М. Некоторые вопросы подобия и факторизации матричных многочленов. Дисс. ... к.ф.--м.н. Львов: ИППММ, 1989.

[11] Крупник И.Н. О разложении матричного пучка на линейные множители // Матем. заметки. 1991. Т. 49. № 2. С. 95-101.

[12] Krupnik I. Decomposition of a monic matrix polynomial into a product of linear factors // Linear Algebra and Appl. 1992. V. 167. P. 239-242.

[13] Икрамов Х. Д. Численное решение матричных уравнений. М.: Наука, 1984.

[14] Шедрик В. П. К вопросу разложимости матричных многочленов на множители. Автореферат дисс. ... к.ф.-м.н. Ленинград: ЛГУ, 1988. 